AperTO - Archivio Istituzionale Open Access dell'Università di Torino

\title{
N-Functionalization of Azoles via Coupling Reactions with Alkoxydienyl and Alkoxystyryl Boronic
} Esters

\section{This is the author's manuscript}

Original Citation:

Availability:

This version is available http://hdl.handle.net/2318/10326

since

Published version:

DOI:10.1002/ejoc.200600872

Terms of use:

Open Access

Anyone can freely access the full text of works made available as "Open Access". Works made available under a Creative Commons license can be used according to the terms and conditions of said license. Use of all other works requires consent of the right holder (author or publisher) if not exempted from copyright protection by the applicable law. 


\title{
$N$-Functionalization of Azoles through Coupling Reactions with Alkoxydienyl and Alkoxystyryl Boronic Esters
}

\author{
Annamaria Deagostino, ${ }^{[a]}$ Cristina Prandi, ${ }^{* a]}$ Chiara Zavattaro, ${ }^{[a]}$ and Paolo Venturello ${ }^{[a]}$
}

Keywords: Azoles / Vinyl boronates / Cross-coupling / C-N bond formation / Copper catalysis

Alkoxydienyl boronates $\mathbf{1 a}$ and $\mathbf{1 b}$ and alkoxystyryl boronate 2 have been used in various copper mediated cross-coupling reactions with azoles. A variety of $\mathrm{N}$-alkoxydienyl- and $\mathrm{N}$ styrylazoles have been synthesized under mild conditions.
The process utilizes $\mathrm{Cu}(\mathrm{OAc})_{2}$ in the presence of $\mathrm{CsF}$ in $\mathrm{CH}_{2} \mathrm{Cl}_{2}$ at room temperature.

() Wiley-VCH Verlag GmbH \& Co. KGaA, 69451 Weinheim, Germany, 2007)

\section{Introduction}

$N$-Functionalized aromatic azacycles are important compounds in naturally occurring products, as well as in many biologically active pharmaceuticals. ${ }^{[1]}$ Transition metalcatalyzed cross-coupling reactions are well established for $\mathrm{C}-\mathrm{N}$ bond formation, but examples involving heterocycles remain rare in these experiments. ${ }^{[2]}$ Traditionally, Ullmanntype coupling between aryl halides and $\mathrm{N}$-containing heterocycles represents a straightforward and inexpensive method by which to arylate azacycles, but the scope of the reaction is limited by the high temperatures required, by the use of 2 equiv or more of aryl halide necessary to provide satisfactory yields, and by the poor tolerance for several important functional groups. ${ }^{[3]}$ Although Pd-catalyzed arylation of azoles is well documented, the scope of this reaction is restricted by $C$-arylation side reactions and by the high cost of the catalyst. ${ }^{[4]} \mathrm{Cu}$-mediated $\mathrm{N}$-arylation of imidazoles was proposed by Buchwald, ${ }^{[5]}$ who reported that the coupling reaction proceeds fairly smoothly in the presence of $\mathrm{Cu}(\mathrm{OTf})_{2}$. benzene complex as a $\mathrm{Cu}$ source and 1,10-phenanthroline as a ligand. Moreover, simple diamine ligands for $\mathrm{CuI}$ have also been used for the arylation of indoles, ${ }^{[6]}$ pyrroles, pyrazoles, indazoles, imidazoles, and triazoles. ${ }^{[7]}$ Recent developments reported by Chan and Lam $^{[8]}$ have demonstrated that arylboronic acids can be effective as arylating agents when stoichiometric quantities of $\mathrm{Cu}(\mathrm{OAc})_{2}$ are used; these experimental conditions have been applied to the arylation of azoles, ${ }^{[9]}$ pyrrole and methyl indole-2-carboxylate derivatives, ${ }^{[10]}$ sterically hindered imidazoles, ${ }^{[11]}$ and electron-deficient pyrroles. ${ }^{[12]}$ More recently, heteroarenes have been arylated with catalytic $\mathrm{Cu}(\mathrm{OAc})_{2}$ in the presence of stoichiometric amounts of mild oxidizing agents, ${ }^{[13]}$ while Collman ${ }^{[14]}$ has reported the arylation of

[a] Dipartimento di Chimica Generale ed Organica Applicata dell'Università,

Via P. Giuria 7, 10125 Torino, Italy

E-mail: cristina.prandi@unito.it imidazoles in the presence of catalytic $[\mathrm{Cu}(\mathrm{OH})$. TMEDA $]_{2} \mathrm{Cl}_{2}$.

In contrast, there are fewer examples of $\mathrm{C}-\mathrm{N}$ bond formation as a method for $N$-vinylation through the use of boronic acids or esters. Lam $^{[15]}$ reported the vinylation of benzimidazole and indazole with either stoichiometric or catalytic $\mathrm{Cu}(\mathrm{OAc})_{2}$ in the presence of oxidants, while $\mathrm{N}$ vinylation of aziridines with alkenylboronic acid was achieved by Yudin. ${ }^{[16]}$ In addition, $N$-vinylation has also been achieved by coupling between vinyl bromides and lithiated azoles ${ }^{[17]}$ or, more recently, azoles in the presence of catalytic $\mathrm{Cu}^{\left[{ }^{[18]}\right.} \mathrm{Pd}$-catalyzed reactions with vinyl triflates have been also reported. ${ }^{[19]}$ In the past few years we have been developing a general method for the synthesis of alkoxydienyl and alkoxystyryl boronates, ${ }^{[20]}$ which have proven to be useful substrates in Suzuki-Miyaura crosscoupling reactions. Here we report the extension of the synthetic applications of this class of vinyl boronates to the formation of $\mathrm{C}-\mathrm{N}$ bonds.

\section{Results and Discussion}

Boronates 1a, 1b, and 2 (Figure 1) were synthesized starting from 1,1-diethoxybut-2-ene, 1,1-diethoxy-3-methylbut2-ene, and 1-(2,2-dimethoxyethyl)benzene, respectively, ${ }^{[20]}$ in the presence of 2.5 equiv. of $\mathrm{LIC} / \mathrm{KOR}$ base $(\mathrm{LIC}=\mathrm{BuLi}$ and $\mathrm{KOR}=t \mathrm{BuOK}) \cdot{ }^{[21]}$ The boronates were isolated as 2,2dimethylpropane-1,3-diol derivatives and, in the cases of 1a and $\mathbf{2}$, as pure $(E)$ isomers. Imidazole was found to provide
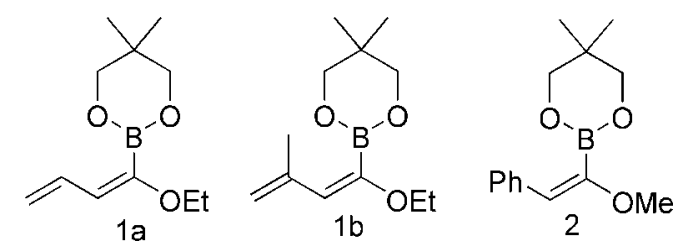

Figure 1. Boronic esters used in the $\mathrm{C}-\mathrm{N}$ cross-coupling. 
good reactivity in the coupling reaction, and was consequently chosen as a substrate for a series of screening experiments.

The experimental conditions developed by Chan and Lam $^{[22]}$ necessitate an excess of boronic acid. Because of the commercial unavailability of the boronic esters $\mathbf{1 a}, \mathbf{1} \mathbf{b}$, and $\mathbf{2}$ we were interested in developing coupling conditions that could permit the use of no more than 1.0 equiv. of the boronate. For this purpose, the initially chosen reaction conditions were the same as those described by Chan and Lam, except for the concentration of the boronate:[8a] imidazole (1.0 equiv.), $\mathrm{Cu}(\mathrm{OAc})_{2} \quad$ (1.5 equiv.), and $\mathrm{Et}_{3} \mathrm{~N}$ (1.0 equiv.) were stirred in $\mathrm{CH}_{2} \mathrm{Cl}_{2}$ at room temperature for 10 min. A solution of $1 \mathrm{a}$ (1.0 equiv.) in $\mathrm{CH}_{2} \mathrm{Cl}_{2}$ was added and the mixture was stirred at room temperature to provide (E)-1-(1-ethoxybuta-1,3-dienyl)-1 $H$-imidazole in 38\% yield (Table 1, Entry 1), but incomplete conversion was observed. A search for other possible catalysts and conditions for this vinylation chemistry was therefore undertaken, and initially we observed that the use of 0.3 equiv. $\mathrm{Cu}(\mathrm{OAc})_{2}$ instead of 1.0 equiv. or more (Entry 2) in an open air vessel slightly improved the yield. Such an enhancement of the yield obtained with a smaller amount of $\mathrm{Cu}$ source is probably due to the fact that the acetate anion, in addition to being a ligand, can also act as a nucleophile, a hypothesis supported by the isolation of 1-ethoxybuta-1,3-dienyl acetate as a byproduct when an excess of $\mathrm{Cu}(\mathrm{OAc})_{2}$ was used (Entry 1). We also tried to operate with catalytic quantities of $\mathrm{Cu}(\mathrm{OAc})_{2}$ (Entries 3 and 4) in the presence of equimolar amounts of mild oxidizing agents. Unfortunately, competitive oxidation of the boronic esters also occurred, ${ }^{[13]}$ and no appreciable amount of the expected coupling product was obtained. It was also found (Entry 5) that switching to a less polar solvent, such as toluene, resulted in a poorer yield. In addition, we examined the coupling between imid- azole and 1a both with $\mathrm{CuI}$ in the presence of 1,10-phenanthroline as a ligand ${ }^{[23]}$ (Entry 6) and with $\mathrm{Cu}(\mathrm{OAc})_{2} \cdot \mathrm{H}_{2} \mathrm{O}$ $10 \%$ (Entry 7 ). ${ }^{[24]}$ In both cases very poor levels of conversion were achieved.

The role of the base was also investigated. In all probability the basic medium plays a double function, both deprotonating the heterocycle- $\mathrm{Cu}^{\mathrm{II}}$ complex and acting as a ligand for the $\mathrm{Cu}$ intermediate. While in the cases of $\mathrm{Cs}_{2} \mathrm{CO}_{3}$ and $t \mathrm{BuOK}$ the role as base prevails, $\mathrm{Et}_{3} \mathrm{~N}$, pyridine, and DMAP could reasonably be effective ligands. The results obtained are reported in Table 1 (Entries 8-12): higher yields were obtained with $\mathrm{Cs}_{2} \mathrm{CO}_{3}$ and $t \mathrm{BuOK}$. A remarkable amount of the homocoupling product derived from 1a was observed when pyridine was used as a base (Entry 10). Moreover, we speculated that the presence of fluoride anions might increase the rate of the transmetalation step, and to our delight the combination of $t \mathrm{BuOK}$ (1.0 equiv.) and CsF (1.0 equiv.) afforded an $88 \%$ yield (Entry 12$)$. Consequently, in a typical procedure, $\mathrm{Cu}(\mathrm{OAc})_{2}$ ( 0.3 equiv.), freshly sublimated $t \mathrm{BuOK}$ (1.0 equiv.), $\mathrm{CsF}$ (1.0 equiv.), and imidazole (1.0 equiv.) in $\mathrm{CH}_{2} \mathrm{Cl}_{2}$ were stirred in a vessel open to air. Dienylboronate (1.0 equiv.) in $\mathrm{CH}_{2} \mathrm{Cl}_{2}$ was then added dropwise and the reaction progress was monitored by TLC. After $1 \mathrm{~h}$ at room temperature the boronate had been consumed, and the crude reaction product was elaborated by addition of a solution of ammonia $(10 \%)$ in saturated aqueous $\mathrm{NH}_{4} \mathrm{Cl}$ to free the heterocycles from $\mathrm{Cu}$ salts. We also investigated the effects of molecular sieves, and found that they do not improve the reaction yield (Entry 13). ${ }^{[14 a]}$

In view of the results obtained for $1 \mathrm{H}$-imidazole, the $\mathrm{N}$ vinylation reactions of pyrazole, indazole, benzimidazole, and pyrrole derivatives were also examined (Table 2). In general, more nucleophilic heterocycles undergo coupling with fairly good yields (Table 2, Entries 1-4). Moreover,

Table 1. Optimization for $N$-vinylation of imidazole. ${ }^{[a]}$

\begin{tabular}{|c|c|c|c|c|c|}
\hline Entry & Solvent & Base & Catalyst & $T$ & Yield $[\%]^{[b]}$ \\
\hline 1 & DCM & $\mathrm{Et}_{3} \mathrm{~N}$ & $\mathrm{Cu}(\mathrm{OAc})_{2} 1.0$ equiv. & room temp. & 38 \\
\hline 2 & DCM & $\mathrm{Et}_{3} \mathrm{~N}$ & $\mathrm{Cu}(\mathrm{OAc})_{2} 0.3$ equiv. & room temp. & 52 \\
\hline 3 & DCM & $\mathrm{Et}_{3} \mathrm{~N}$ & $\mathrm{Cu}(\mathrm{OAc})_{2} 0.05$ equiv., TEMPO & room temp. & - \\
\hline 4 & $\mathrm{DCM}$ & $\mathrm{Et}_{3} \mathrm{~N}$ & $\mathrm{Cu}(\mathrm{OAc})_{2} 0.05$ equiv., $\mathrm{NMO}$ & room temp. & - \\
\hline 5 & $\mathrm{PhMe}$ & $\mathrm{Et}_{3} \mathrm{~N}$ & $\mathrm{Cu}(\mathrm{OAc})_{2} 0.3$ equiv. & room temp. & 25 \\
\hline 6 & DCM & $\mathrm{Cs}_{2} \mathrm{CO}_{3}$ & $\mathrm{CuI} / 1,10$-phenanthroline & room temp. & 15 \\
\hline 7 & DCM & $\mathrm{Cs}_{2} \mathrm{CO}_{3}$ & $\mathrm{Cu}(\mathrm{OAc})_{2} \cdot \mathrm{H}_{2} \mathrm{O}$ & room temp. & 11 \\
\hline 8 & DCM & $\mathrm{Cs}_{2} \mathrm{CO}_{3}$ & $\mathrm{Cu}(\mathrm{OAc})_{2} 0.3$ equiv. & room temp. & 58 \\
\hline 9 & DCM & DMAP & $\mathrm{Cu}(\mathrm{OAc})_{2} 0.3$ equiv. & room temp. & 30 \\
\hline 10 & DCM & pyridine & $\mathrm{Cu}(\mathrm{OAc})_{2} 0.3$ equiv. & room temp. & 40 \\
\hline 11 & $\mathrm{DCM}$ & $t \mathrm{BuOK}$ & $\mathrm{Cu}(\mathrm{OAc})_{2} 0.3$ equiv. & room temp. & 62 \\
\hline 12 & DCM & $t \mathrm{BuOK} / \mathrm{CsF}$ & $\mathrm{Cu}(\mathrm{OAc})_{2} 0.3$ equiv. & room temp. & 88 \\
\hline 13 & DCM & $t \mathrm{BuOK} / \mathrm{CsF}$ (mol. sieves, $4 \AA$ ) & $\mathrm{Cu}(\mathrm{OAc})_{2} 0.3$ equiv. & room temp. & 75 \\
\hline
\end{tabular}

[a] Reaction conditions: 1a $(1.0 \mathrm{mmol})$, solvent $(5 \mathrm{~mL})$. [b] Average of two runs, refers to products isolated by flash chromatography on $\mathrm{SiO}_{2}$, estimated to be $>97 \%$ pure by ${ }^{1} \mathrm{H}$ and ${ }^{13} \mathrm{C}$ NMR analyses. 
Table 2. Functionalized azoles obtained by cross-coupling of alkoxyboronates $\mathbf{1 a}, \mathbf{1 b}$, and $\mathbf{2}$ with diverse N-H-containing heteroarenes. ${ }^{[a]}$

\begin{tabular}{|c|c|c|c|c|c|c|c|c|}
\hline Entry & Product & $\begin{array}{l}\text { Yield } \\
(\%)^{[b]}\end{array}$ & Entry & Product & $\begin{array}{l}\text { Yield } \\
(\%)^{[\mathrm{b}]}\end{array}$ & Entry & Product & $\begin{array}{l}\text { Yield } \\
(\%)^{[b]}\end{array}$ \\
\hline 1 & $\mathbf{a}$ & 88 & 7 & & 85 & 13 & $3 k$ & 89 \\
\hline 2 & b & 78 & $8^{[\mathrm{f}]}$ & & 58 & 14 & 31 & 71 \\
\hline 3 & & 65 & 9 & & 76 & 15 & & 65 \\
\hline $4^{[c]}$ & & 58 & 10 & & traces & $16^{[\mathrm{f}]}$ & & 60 \\
\hline $5^{[\mathrm{d}]}$ & e & 21 & 11 & & n.d. & 17 & & 83 \\
\hline $6^{[\mathrm{e}]}$ & & $<5 \%$ & 12 & & 43 & & & \\
\hline
\end{tabular}

[a] Reaction conditions: boronate (1.0 equiv.), heteroarene (1.0 equiv.), base (1.0 equiv.), $\mathrm{CsF}(1.0$ equiv. $), \mathrm{Cu}(\mathrm{OAc})_{2}\left(0.3\right.$ equiv.), $\mathrm{CH}_{2} \mathrm{Cl}_{2}$ $(5.0 \mathrm{~mL})$. Spectral data are in accordance with the assigned structures. [b] Products purified by flash chromatography. [c] Two isomers were detected in a 3:1 ratio $\left({ }^{1} \mathrm{H}\right.$ NMR integration). [d] Different conditions were tested to improve the yield: Py and Et ${ }_{3} \mathrm{~N}$ do not promote the reaction. Compound 3e proved to be very unstable; it has been identified only by ${ }^{1} \mathrm{H}$ NMR and GC-MS. [e] Product detected by GC-MS and ${ }^{1} \mathrm{H}$ NMR analyses, after flash chromatography purification. [f] Reaction conducted without base.

only small amounts of the desired products were isolated from the complex reaction mixture in the cases of pyrrole and indole (Entries 5-6); similar results have been reported previously. ${ }^{\left[{ }^{[]}\right.}$From the enhanced reactivity of electron-deficient azoles, we assumed that electron-deficient pyrrole and indole derivatives might exhibit higher reactivities, and to evaluate this hypothesis a variety of electron-deficient substrates were examined. Pyrrole-2-carbaldehyde, 1-(pyrrol-2-yl)ethanone, and methyl pyrrole-2-carboxylate underwent coupling reactions under the aforementioned experimental conditions, affording the corresponding coupled products in good yields (Entries 7-9). Furthermore, in the case of 1-pyrrol-2-ylethanone the initially obtained yields were unsatisfactory, probably as a consequence of the fact that a strongly basic medium ( $t \mathrm{BuOK} 1.0$ equiv.) is not compatible with the enolizable acetyl group.

The reaction was then conducted in the absence of any base. Even under these conditions, however, alkoxydienyl dimer $(15 \%)$ was also recovered along with the desired product. As far as indole derivatives are concerned, the presence of an electron-withdrawing substituent in position 2 gave rise to an acceptable yield of $N$-functionalized derivative (Entry 12): in contrast, very little conversion was observed in the cases of unsubstituted indole (Entry 6) or indoles substituted in position 3 (Entries 10 and 11). The experimental conditions that we had established were then applied to boronates $\mathbf{1 b}$ and $\mathbf{2}$ (Table 2, Entries 13 and 14, and $15-17$, respectively). In all cases the reaction yields obtained were satisfactory and confirm the general applicability of the proposed method. In the case of boronate $\mathbf{1 b}$, the elimination process lacks complete $(E)$ stereoselectivity, the $(Z)$ minor isomer also being obtained $(10 \%)$, as a consequence of which the coupling products $3 \mathbf{k}$ and $\mathbf{3 l}$ were recovered as mixtures of $(E)$ and $(Z)$ isomers in 9:1 ratios, as deduced from their ${ }^{1} \mathrm{H}$ NMR spectra. Moreover, methyl 1(1-methoxy-2-phenylvinyl)-1H-pyrrole-2-carboxylate (3o) was isolated by flash chromatography, and its stereochemistry was confirmed by ROESY experiments. It is noteworthy 
that the optimized experimental conditions are well matched with useful functional groups that could be subjected to further synthetic elaboration.

\section{Conclusions}

In summary, we have explored the reactivity of alkoxydienyl- and alkoxystyrylboronic esters in $\mathrm{C}-\mathrm{N}$ cross-coupling reactions with various azoles. The good yields and the mild conditions of this methodology make it a promising method for $N$-functionalization of heterocycles. We are currently exploring the experimental conditions to perform the unmasking reaction of the vinyl ether functionality in order to expand the scope of this synthetic sequence.

\section{Experimental Section}

Chromatographic separations were carried out on silica gel by flash column techniques; $R_{\mathrm{f}}$ values refer to TLC carried out on $0.25 \mathrm{~mm}$ silica gel plates (Merck F254), with the same eluent as indicated for the column chromatography. ${ }^{1} \mathrm{H}$ NMR spectra and NOESY $2 \mathrm{D}$ experiments were recorded at $200 \mathrm{MHz},{ }^{13} \mathrm{C}$ NMR spectra at $50.33 \mathrm{MHz}$. MS spectra were recorded at an ionizing voltage of $70 \mathrm{eV}$. THF was distilled from Na/benzophenone. Compounds 1a, $\mathbf{1 b}$, and $\mathbf{2}^{[19]}$ were prepared as reported.

Cross-Coupling Reactions. General Procedure: $\mathrm{Cu}(\mathrm{OAc})_{2}(0.3 \mathrm{mmol}$, 0.3 equiv., $54 \mathrm{mg}$ ), freshly sublimated $t \mathrm{BuOK}$ (1.0 equiv., $112 \mathrm{mg}$ ), CsF (1.0 equiv., $152 \mathrm{mg}$ ), and the corresponding azole (1.0 equiv.) in DCM $(5 \mathrm{~mL})$ were stirred in a vessel open to air. The boronate (1.0 equiv.), dissolved in $\mathrm{CH}_{2} \mathrm{Cl}_{2}(2 \mathrm{~mL})$, was then added dropwise and the reaction progress was monitored by TLC. After $1 \mathrm{~h}$ at room temperature the boronate had usually been completely consumed. The crude reaction product was elaborated by addition of a solution of ammonia in saturated $\mathrm{NH}_{4} \mathrm{Cl}(10 \%)$ to free the heterocycles from copper salts. The mixture was then extracted with DCM $(3 \times 20 \mathrm{~mL})$ and dried with anhydrous $\mathrm{K}_{2} \mathrm{CO}_{3}$. Evaporation of the solvent afforded a green-yellow oil, which was purified by flash chromatography (EtOAc/petroleum ether, 1:4, 1\% $\mathrm{Et}_{3} \mathrm{~N}$ ).

(E)-1-(1-Ethoxybuta-1,3-dienyl)-1 $\boldsymbol{H}$-imidazole (3a): This compound (144 mg, 88\%) was obtained as a colorless oil: ${ }^{1} \mathrm{H}$ NMR $(200 \mathrm{MHz}$, $\left.\mathrm{CDCl}_{3}, \mathrm{TMS}\right): \delta=7.65$ (s, $\left.1 \mathrm{H}\right), 7.10(\mathrm{~s}, 2 \mathrm{H}), 6.15(\mathrm{dt}, J=16.1$, $10.2 \mathrm{~Hz}, 1 \mathrm{H}), 5.34(\mathrm{~d}, J=10.2 \mathrm{~Hz}, 1 \mathrm{H}), 5.15(\mathrm{dd}, J=16.1,1.5 \mathrm{~Hz}$, $1 \mathrm{H}), 4.95(\mathrm{dd}, J=10.2,1.5 \mathrm{~Hz}, 1 \mathrm{H}), 3.85(\mathrm{q}, J=6.5 \mathrm{~Hz}, 2 \mathrm{H}), 1.36$ (t, $J=6.5 \mathrm{~Hz}, 3 \mathrm{H}) \mathrm{ppm} .{ }^{13} \mathrm{C} \mathrm{NMR}\left(50.33 \mathrm{MHz}, \mathrm{CDCl}_{3}\right.$, TMS): $\delta=146.06,137.29,129.98,128.98,119.14,115.09,97.88,64.79$, 14.28 ppm. MS (E/I): $m / z(\%)=164(29)\left[\mathrm{M}^{+}, 135(100), 107\right.$ (45), 68 (75). $\mathrm{C}_{9} \mathrm{H}_{12} \mathrm{~N}_{2} \mathrm{O}: \mathrm{C} 65.83, \mathrm{H} 7.37, \mathrm{~N} 17.06$; found $\mathrm{C} 65.79, \mathrm{H}$ 7.73, N 16.85 .

(E)-1-(1-Ethoxybuta-1,3-dienyl)-1 $\boldsymbol{H}$-pyrazole (3b): This compound (128 mg, 78\%) was obtained as a colorless oil: ${ }^{1} \mathrm{H}$ NMR $(200 \mathrm{MHz}$, $\left.\mathrm{CDCl}_{3}, \mathrm{TMS}\right): \delta=7.75(\mathrm{~d}, J=5.2 \mathrm{~Hz}, 2 \mathrm{H}), 6.55(\mathrm{dt}, J=16.2$, $10.1 \mathrm{~Hz}, 1 \mathrm{H}), 6.32(\mathrm{t}, J=5.2 \mathrm{~Hz}, 1 \mathrm{H}), 5.44(\mathrm{~d}, J=10.1 \mathrm{~Hz}, 1 \mathrm{H})$, $5.15(\mathrm{dd}, J=16.2,1.5 \mathrm{~Hz}, 1 \mathrm{H}), 4.95(\mathrm{dd}, J=10.1,1.5 \mathrm{~Hz}, 1 \mathrm{H})$, 3.95 (q, $J=6.8 \mathrm{~Hz}, 2 \mathrm{H}), 1.46(\mathrm{t}, J=6.8 \mathrm{~Hz}, 3 \mathrm{H}) \mathrm{ppm} .{ }^{13} \mathrm{C} \mathrm{NMR}$ (50.33 MHz, $\left.\mathrm{CDCl}_{3}, \mathrm{TMS}\right): \delta=146.55,140.91,130.96,130.24$, $114.54,106.12,97.18,64.81,14.30$ (q) ppm. MS (E/I): $m / z(\%)=$ 164 (12) $[\mathrm{M}]^{+}, 135$ (100), 107 (35), 68 (67). $\mathrm{C}_{9} \mathrm{H}_{12} \mathrm{~N}_{2} \mathrm{O}: \mathrm{C}$ 65.83, H 7.37, N 17.06; found C 65.89, H 7.41, N 16.98.
(E)-1-(1-Ethoxybuta-1,3-dienyl)-1 $\boldsymbol{H}$-benzo[d] imidazole $\quad(3 \mathrm{c})$ : This compound (139 mg, 65\%) was obtained as a pale yellow oil: ${ }^{1} \mathrm{H}$ NMR (200 MHz, $\left.\mathrm{CDCl}_{3}, \mathrm{TMS}\right): \delta=8.01$ (s, $\left.1 \mathrm{H}\right), 7.75$ (d, $J=$ $7.8 \mathrm{~Hz}, 2 \mathrm{H}), 7.40$ (d, $J=7.8 \mathrm{~Hz}, 2 \mathrm{H}), 6.00(\mathrm{dt}, J=16.3,10.1 \mathrm{~Hz}$, $1 \mathrm{H}), 5.65(\mathrm{~d}, J=10.1 \mathrm{~Hz}, 1 \mathrm{H}), 5.15(\mathrm{dd}, J=16.3,1.5 \mathrm{~Hz}, 1 \mathrm{H})$, $4.85(\mathrm{dd}, J=10.1,1.5 \mathrm{~Hz}, 1 \mathrm{H}), 4.05(\mathrm{q}, J=6.5 \mathrm{~Hz}, 2 \mathrm{H}), 1.45(\mathrm{t}$, $J=6.5 \mathrm{~Hz}, 3 \mathrm{H}) \mathrm{ppm} .{ }^{13} \mathrm{C} \mathrm{NMR}\left(50.33 \mathrm{MHz}, \mathrm{CDCl}_{3}, \mathrm{TMS}\right): \delta=$ $147.35,139.88,135.96,130.95,127.12,123.88,121.55,120.78$, $114.36,110.88,100.39,64.66,14.23 \mathrm{ppm}$. MS (E/I): $m / z(\%)=214$ (5) $[\mathrm{M}]^{+}, 185$ (100), 169 (25), 118 (94), 68 (12). $\mathrm{C}_{13} \mathrm{H}_{14} \mathrm{~N}_{2} \mathrm{O}: \mathrm{C}$ 72.87, H 6.59, N 13.07; found C 72.51, H 6.55, N 13.15 .

(E)-1-(1-Ethoxybuta-1,3-dienyl)-1 $\boldsymbol{H}$-indazole (3d): A mixture of two diastereoisomers $(124 \mathrm{mg}, 58 \%)$ was obtained as a pale yellow oil: ${ }^{1} \mathrm{H}$ NMR (200 MHz, $\mathrm{CDCl}_{3}$, TMS) major isomer: $\delta=8.01(\mathrm{~s}, 1$ $\mathrm{H}), 7.80-7.75(\mathrm{~m}, 2 \mathrm{H}), 7.35-7.29(\mathrm{~m}, 2 \mathrm{H}), 5.95(\mathrm{dt}, J=16.4$, $10.0 \mathrm{~Hz}, 1 \mathrm{H}), 5.64(\mathrm{~d}, J=10.0 \mathrm{~Hz}, 1 \mathrm{H}), 5.20(\mathrm{dd}, J=16.4,1.5 \mathrm{~Hz}$, $1 \mathrm{H}), 4.95$ (dd, $J=10.0,1.5 \mathrm{~Hz}, 1 \mathrm{H}), 3.95$ (q, $J=6.5 \mathrm{~Hz}, 2 \mathrm{H}), 1.46$ $(\mathrm{t}, J=6.5 \mathrm{~Hz}, 3 \mathrm{H}) \mathrm{ppm} .{ }^{13} \mathrm{C} \mathrm{NMR}\left(50.33 \mathrm{MHz}, \mathrm{CDCl}_{3}, \mathrm{TMS}\right): \delta$ $=148.22,140.75,136.83,131.82,127.99,124.75,122.43,121.65$, $115.23,111.75,101.26,65.54,15.11 \mathrm{ppm}$. MS (E/I): $m / z(\%)=214$ (86) $[\mathrm{M}]^{+}, 185$ (100), 157 (77), 119 (44), 69 (42). $\mathrm{C}_{13} \mathrm{H}_{14} \mathrm{~N}_{2} \mathrm{O}: \mathrm{C}$ 72.87, H 6.59, N 13.07; found C 72.62, H 6.62, N 13.12.

(E)-1-(1-Ethoxybuta-1,3-dienyl)-1H-pyrrole (3e): This compound (34 mg, 21\%) was obtained as a pale yellow oil: ${ }^{1} \mathrm{H}$ NMR (200 MHz, $\mathrm{CDCl}_{3}$, TMS) major isomer: $\delta=6.85-6.79(\mathrm{~m}, 2 \mathrm{H})$, 6.25-6.19 (m, $2 \mathrm{H}$ superimposed to 6.25 ; dt, $J=16.1,10.1 \mathrm{~Hz}, 1$ $\mathrm{H}), 5.25(\mathrm{~d}, J=10.1 \mathrm{~Hz}, 1 \mathrm{H}), 5.10(\mathrm{dd}, J=16.1,1.5 \mathrm{~Hz}, 1 \mathrm{H})$, $4.85(\mathrm{dd}, J=10.1,1.5 \mathrm{~Hz}, 1 \mathrm{H}), 3.95(\mathrm{q}, J=6.8 \mathrm{~Hz}, 2 \mathrm{H}), 1.40(\mathrm{t}$, $J=6.8 \mathrm{~Hz}, 3 \mathrm{H}) \mathrm{ppm}$. MS (E/I): $m / z(\%)=163(26)[\mathrm{M}]^{+}, 134$ (100), 106 (44), 68 (66).

(E)-1-(1-Ethoxybuta-1,3-dienyl)-1 $\boldsymbol{H}$-indole (3f): This compound $(5 \mathrm{mg}, 2.3 \%)$ was obtained as a pale yellow oil: ${ }^{1} \mathrm{H}$ NMR (200 MHz, $\mathrm{CDCl}_{3}$, TMS): $\delta=7.72-7.29$ (m, $\left.2 \mathrm{H}\right), 7.44-7.31$ (m, 4 $\mathrm{H}), 5.95(\mathrm{dt}, J=16.0,10.0 \mathrm{~Hz}, 1 \mathrm{H}), 5.60(\mathrm{~d}, J=10.0 \mathrm{~Hz}, 1 \mathrm{H})$, $5.20(\mathrm{dd}, J=16.0,1.5 \mathrm{~Hz}, 1 \mathrm{H}), 4.95(\mathrm{dd}, J=10.0,1.5 \mathrm{~Hz}, 1 \mathrm{H})$, $4.05(\mathrm{q}, J=6.8 \mathrm{~Hz}, 2 \mathrm{H}), 1.35(\mathrm{t}, J=6.8 \mathrm{~Hz}, 3 \mathrm{H}) \mathrm{ppm}$. MS (E/I): $m / z(\%)=213(10)[\mathrm{M}]^{+}, 116(72), 97(14), 69(44)$.

(E)-1-(1-Ethoxybuta-1,3-dienyl)-1 $H$-pyrrole-2-carbaldehyde $\quad(3 g)$ : This compound $(162 \mathrm{mg}, 85 \%)$ was obtained as a yellow oil: ${ }^{1} \mathrm{H}$ NMR (200 MHz, $\left.\mathrm{CDCl}_{3}, \mathrm{TMS}\right): \delta=9.59$ (s, $\left.1 \mathrm{H}\right), 7.07$ (dd, $J=$ $3.9,1.6 \mathrm{~Hz}, 1 \mathrm{H}), 7.00(\mathrm{brs}, 1 \mathrm{H}), 6.35(\mathrm{dd}, J=3.9,1.6 \mathrm{~Hz}, 1 \mathrm{H})$, $5.83(\mathrm{dt}, J=16.8,10.4 \mathrm{~Hz}, 1 \mathrm{H}), 5.5(\mathrm{~d}, J=10.42 \mathrm{~Hz}, 1 \mathrm{H}), 5.1$ $(\mathrm{dd}, J=16.8,1.7 \mathrm{~Hz}, 1 \mathrm{H}), 4.86(\mathrm{dd}, J=10.4,1.7 \mathrm{~Hz}, 1 \mathrm{H}), 4.02$ (q, $J=7.06, \mathrm{~Hz}, 2 \mathrm{H}), 1.35(\mathrm{t}, J=7.09 \mathrm{~Hz}, 3 \mathrm{H}) \mathrm{ppm} .{ }^{13} \mathrm{C} \mathrm{NMR}$ (50.33 MHz, $\left.\mathrm{CDCl}_{3}, \mathrm{TMS}\right): \delta=178.36,147.81,132.46,130.47$, $130.12,121.57,114.54,110.38,110.60,64.97,14.16$ ppm. MS (EI): $m / z(\%)=191(15)[\mathrm{M}]^{+}, 162(88), 134$ (54), 94 (45), 68 (100). $\mathrm{C}_{11} \mathrm{H}_{13} \mathrm{NO}_{2}$ : C $69.09, \mathrm{H} 6.85, \mathrm{~N} 7.32$; found $\mathrm{C} 69.43, \mathrm{H} 6.35, \mathrm{~N}$ 7.26.

(E)-1-[1-(1-Ethoxybuta-1,3-dienyl)-1 $H$-pyrrol-2-yl]ethanone (3h): This compound $(119 \mathrm{mg}, 58 \%)$ was obtained as a yellow oil: ${ }^{1} \mathrm{H}$ NMR (200 MHz, $\left.\mathrm{CDCl}_{3}, \mathrm{TMS}\right):{ }^{1} \mathrm{H} \mathrm{NMR}\left(200 \mathrm{MHz}, \mathrm{CDCl}_{3}\right.$, TMS): $\delta=6.95-6.54(\mathrm{~m}, 1 \mathrm{H}), 6.85-6.76(\mathrm{~m}, 1 \mathrm{H}), 6.00-5.85(\mathrm{~m}$, $1 \mathrm{H}), 5.71(\mathrm{dt}, J=16.96,10.20, \mathrm{~Hz}, 1 \mathrm{H}), 5.40(\mathrm{~d}, J=10.20 \mathrm{~Hz}, 1$ H), $5.04(\mathrm{dd}, J=16.96,1.81 \mathrm{~Hz}, 1 \mathrm{H}), 4.74(\mathrm{dd}, J=10.20,1.81 \mathrm{~Hz}$, $1 \mathrm{H}), 3.98(\mathrm{q}, J=6.97 \mathrm{~Hz}, 2 \mathrm{H}), 2.38(\mathrm{~s}, 3 \mathrm{H}), 1.29(\mathrm{t}, J=6.97 \mathrm{~Hz}, 3$ $\mathrm{H}) \mathrm{ppm} .{ }^{13} \mathrm{C} \mathrm{NMR}\left(50.33 \mathrm{MHz}, \mathrm{CDCl}_{3}\right.$, TMS): $\delta=186.29,149.75$, $131.63,130.65,130.23,119.46,113.35,109,09,99.69,64.95,26.45$, $14.21 \mathrm{ppm}$. MS (EI): $m / z(\%)=205(14)[\mathrm{M}]^{+}, 134(100), 110(11)$, 94 (78), 68 (59). $\mathrm{C}_{12} \mathrm{H}_{15} \mathrm{NO}_{2}$ : C 70.22, $\mathrm{H} \mathrm{7.37,} \mathrm{N} \mathrm{6.82;} \mathrm{found} \mathrm{C}$ $70.26, \mathrm{H} 7.15, \mathrm{~N} 6.54$. 
Methyl (E)-1-(1-Ethoxybuta-1,3-dienyl)-1 $H$-pyrrole-2-carboxylate (3i): This compound (168 mg, 76\%) was obtained as a yellow oil: ${ }^{1} \mathrm{H}$ NMR (200 MHz, $\mathrm{CDCl}_{3}$, TMS): $\delta=6.94$ (ddd, $J=3.80,1.73$, $0.40 \mathrm{~Hz}, 1 \mathrm{H}), 6.82-6.76(\mathrm{~m}, 1 \mathrm{H}), 6.16(\mathrm{ddd}, J=3.80,1.73$, $0.40 \mathrm{~Hz}, 1 \mathrm{H}), 5.74(\mathrm{td}, J=16.89,10.50 \mathrm{~Hz}, 1 \mathrm{H}), 5.39(\mathrm{~d}, J=$ $10.50 \mathrm{~Hz}, 1 \mathrm{H}), 5.03(\mathrm{dd}, J=16.89,1.84 \mathrm{~Hz}, 1 \mathrm{H}), 4.74(\mathrm{dd}, J=$ 10.50, $1.84 \mathrm{~Hz}, 1 \mathrm{H}), 3.92(\mathrm{q}, J=7.02 \mathrm{~Hz}, 2 \mathrm{H}), 3.72(\mathrm{~s}, 3 \mathrm{H}), 1.26$ (t, $J=7.02 \mathrm{~Hz}, 3 \mathrm{H}) \mathrm{ppm} .{ }^{13} \mathrm{C} \mathrm{NMR}\left(50.33 \mathrm{MHz}, \mathrm{CDCl}_{3}\right.$, TMS): $\delta=159.98,149.08,130.63,129.00,123.25,118.18,113.61,109.05$, $100.19,64.79,51.03,14.22 \mathrm{ppm}$. MS (EI): $\mathrm{m} / \mathrm{z}(\%)=221$ (29) $[\mathrm{M}]^{+}, 192$ (34), 162 (90), 134 (67), 94 (96). $\mathrm{C}_{12} \mathrm{H}_{15} \mathrm{NO}_{3}: \mathrm{C}$ 65.14, $\mathrm{H}$ 6.83, N 6.33; found C 65.26, H 6.75, N 6.24.

Ethyl (E)-1-(1-Ethoxybuta-1,3-dienyl)-1 $\boldsymbol{H}$-indole-2-carboxylate (3j): This compound (122 mg, 43\%) was obtained as a yellow oil. The spectra were recorded after flash chromatography purification and Kugelrohr distillation. ${ }^{1} \mathrm{H}$ NMR $\left(200 \mathrm{MHz}, \mathrm{CDCl}_{3}, \mathrm{TMS}\right): \delta=$ $7.71(\mathrm{~d}, J=6.5 \mathrm{~Hz}, 1 \mathrm{H}), 7.41-7.15(\mathrm{~m}, 4 \mathrm{H}), 5.95(\mathrm{dt}, J=16.0$, $10.0 \mathrm{~Hz}, 1 \mathrm{H}), 5.65(\mathrm{~d}, J=10.0 \mathrm{~Hz}, 1 \mathrm{H}), 5.22(\mathrm{dd}, J=16.0,1.5 \mathrm{~Hz}$, $1 \mathrm{H}), 4.95(\mathrm{dd}, J=10.0,1.5 \mathrm{~Hz}, 1 \mathrm{H}), 4.24(\mathrm{q}, J=6.7,2 \mathrm{H}), 4.04$ $(\mathrm{q}, J=7.0,2 \mathrm{H}), 1.26(\mathrm{t}, J=6.7 \mathrm{~Hz}, 3 \mathrm{H}), 1.15(\mathrm{t}, J=7.0 \mathrm{~Hz}, 3$ H) ppm. ${ }^{13} \mathrm{C} \mathrm{NMR}\left(50.33 \mathrm{MHz}, \mathrm{CDCl}_{3}, \mathrm{TMS}\right): \delta=160.49,146.99$, $138.91,131.11,128.66,126.09,125.67,122.23,121.43,113.69$, $112.07,112.03,101.32,64.61,60.50,14.27,14.12$ ppm. MS (EI): $m / z(\%)=285(16)[\mathrm{M}]^{+}, 212(100), 184(90), 143(50), 115(31)$. $\mathrm{C}_{17} \mathrm{H}_{19} \mathrm{NO}_{3}$ : C 71.56, H 6.71, N 4.91; found C 71.45, H 6.34, N 4.23

(E)-1-(1-Ethoxy-3-methylbuta-1,3-dienyl)-1 $H$-pyrrole-2-carbaldehyde (3k): This compound (182 $\mathrm{mg}, 89 \%)$ was obtained as a yellow oil: ${ }^{1} \mathrm{H}$ NMR (200 MHz, $\mathrm{CDCl}_{3}$, TMS): major diastereoisomer: $\delta=9.74(\mathrm{~s}, 1 \mathrm{H}), 7.03(\mathrm{dd}, J=3.85,2.66 \mathrm{~Hz}, 1 \mathrm{H}), 6.94-6.56$ $(\mathrm{m}, 1 \mathrm{H}), 6.30(\mathrm{dd}, J=3.85,2.66 \mathrm{~Hz}, 1 \mathrm{H}), 5.47(\mathrm{~s}, 1 \mathrm{H}), 4.73$ (s, $1 \mathrm{H}), 4.67(\mathrm{~s}, 1 \mathrm{H}), 3.96(\mathrm{q}, J=7.02 \mathrm{~Hz}, 2 \mathrm{H}), 1.35(\mathrm{~s}, 3 \mathrm{H}), 1.31$ (t, $J=7.02 \mathrm{~Hz}, 3 \mathrm{H}) \mathrm{ppm} .{ }^{13} \mathrm{C} \mathrm{NMR}\left(50.33 \mathrm{MHz}, \mathrm{CDCl}_{3}, \mathrm{TMS}\right)$ : $\delta=178.38,146.24,138.55,132.58,130.81,121.85,114.96,110.35$, $102.77,65.22,19.90,14.26 \mathrm{ppm}$. MS (EI): $\mathrm{m} / z(\%)=205(11)$ $[\mathrm{M}]^{+}, 148$ (100), 120 (38), 82 (78). $\mathrm{C}_{12} \mathrm{H}_{15} \mathrm{NO}_{2}$ : C 70.22, H 7.37, N 6.82; found $\mathrm{C} 70.56, \mathrm{H} 7.36, \mathrm{~N} 6.34$.

(E)-1-(1-Ethoxy-3-methylbuta-1,3-dienyl)-1 $\boldsymbol{H}$-benzo $[\boldsymbol{d}]$ imidazole (3l): This compound (162 mg, 71\%) was obtained as a pale yellow oil: ${ }^{1} \mathrm{H}$ NMR (200 MHz, $\mathrm{CDCl}_{3}$, TMS): major diastereoisomer: $\delta$ $=8.16(\mathrm{~s}, 1 \mathrm{H}), 7.74(\mathrm{~d}, J=8.08 \mathrm{~Hz}, 1 \mathrm{H}), 7.42-7.34(\mathrm{~m}, 2 \mathrm{H})$, 7.27-7.15 (m, 1 H), $5.74(\mathrm{~s}, 1 \mathrm{H}), 4.81(\mathrm{~s}, 1 \mathrm{H}), 4.74(\mathrm{~s}, 1 \mathrm{H}), 3.92$ $(\mathrm{q}, J=7.00,2 \mathrm{H}), 1.35(\mathrm{t}, J=7.00 \mathrm{~Hz}, 3 \mathrm{H}), 1.18(\mathrm{~s}, 3 \mathrm{H}) \mathrm{ppm}$. ${ }^{13} \mathrm{C} \mathrm{NMR}\left(50.33 \mathrm{MHz}, \mathrm{CDCl}_{3}, \mathrm{TMS}\right): \delta=145.37,140.44,138.21$, $135.73,127.13,123.75,121.47,120.73,115.63,110.17,104.70$, $64.50,20.06,14.27 \mathrm{ppm}$. MS (EI): $m / z(\%)=228(1)[\mathrm{M}]^{+}, 199$ (100), 183 (28), 118 (40). $\mathrm{C}_{14} \mathrm{H}_{16} \mathrm{NO}_{2}$ : C 73.66, H 7.06, N 12.27; found $\mathrm{C} 73.54, \mathrm{H} 7.76, \mathrm{~N} 12.76$.

(E)-1-(1-Methoxy-2-phenylvinyl)-1 $\boldsymbol{H}$-pyrrole-2-carbaldehyde (3m): This compound $(147 \mathrm{mg}, 65 \%)$ was obtained as a yellow oil: ${ }^{1} \mathrm{H}$ NMR (200 MHz, $\mathrm{CDCl}_{3}$, TMS): $\delta=9.62(\mathrm{~s}, 1 \mathrm{H}), 7.19-7.05(\mathrm{~m}$, $4+1 \mathrm{H}), 6.86(\mathrm{ddd}, J=2.55,1.59,0.76 \mathrm{~Hz}, 1 \mathrm{H}), 6.65(\mathrm{dd}, J=$ 2.05, $0.42 \mathrm{~Hz}, 1 \mathrm{H}), 6.63-6.60(\mathrm{~m}, 1 \mathrm{H}), 6.32(\mathrm{dd}, J=3.92,2.55 \mathrm{~Hz}$, $1 \mathrm{H}), 5.79(\mathrm{~s}, 1 \mathrm{H}), 3.89(\mathrm{~s}, 3 \mathrm{H}) \mathrm{ppm} .{ }^{13} \mathrm{C} \mathrm{NMR}(50.33 \mathrm{MHz}$, $\mathrm{CDCl}_{3}$, TMS): $\delta=178.36,147.72,133.54,131.67,129.77,128.22$, 127.02, 126.08, 122.28, 111.23, 99.31, $56.54 \mathrm{ppm}$. MS (EI): $\mathrm{m} / z(\%)$ $=227(37)[\mathrm{M}]^{+}, 212(57), 183(11), 94(100) . \mathrm{C}_{14} \mathrm{H}_{13} \mathrm{NO}_{2}: \mathrm{C} 73.99$, H 5.77, N 6.16; found C 73.23, H 5.14, N 6.34.

(E)-1-[1-(1-Methoxy-2-phenylvinyl)-1 H-pyrrol-2-yl]ethanone (3n): This compound (145 mg, 60\%) was obtained as a yellow oil: ${ }^{1} \mathrm{H}$ NMR (200 MHz, $\mathrm{CDCl}_{3}, \mathrm{TMS}$ ): $\delta=7.15-7.00$ (m, $4 \mathrm{H}$ ), 6.77 (dd, $J=2.67,1.80 \mathrm{~Hz}, 1 \mathrm{H}), 6.67(\mathrm{dd}, J=1.80,0.55 \mathrm{~Hz}, 1 \mathrm{H}), 6.60$
6.55 (m, 1 H), 6.31-6.14 (m, $1 \mathrm{H}), 5.70$ (s, $1 \mathrm{H}), 3.88$ (s, $3 \mathrm{H}), 2.44$ (s, $3 \mathrm{H}$ ) ppm. ${ }^{13} \mathrm{C}$ NMR (50.33 MHz, $\left.\mathrm{CDCl}_{3}, \mathrm{TMS}\right): \delta=186.85$, $149.65,134.39,131.23,129.54,128.25,127.13,125.80,120.09$, $110.17,98.47,56.79,26.65 \mathrm{ppm}$. MS (EI): $\mathrm{m} / \mathrm{z}(\%)=241(82)$ $[\mathrm{M}]^{+}, 226$ (96), 156 (100), 89 (46). $\mathrm{C}_{15} \mathrm{H}_{15} \mathrm{NO}_{2}$ : C 74.67, H 6.27, N 6.16; found C 73.23, H 5.14, N 5.81.

(E)-Methyl 1-(1-Methoxy-2-phenylvinyl)-1 $\boldsymbol{H}$-pyrrole-2-carboxylate (3o): This compound ( $213 \mathrm{mg}, 83 \%$ ) was obtained as a yellow oil: ${ }^{1} \mathrm{H}$ NMR (200 MHz, $\left.\mathrm{CDCl}_{3}, \mathrm{TMS}\right): \delta=7.25-6.96$ (m, $\left.4 \mathrm{H}\right), 6.76$ $(\mathrm{dd}, J=2.75,1.74 \mathrm{~Hz}, 1 \mathrm{H}), 6.66(\mathrm{dd}, J=1.74,0.52 \mathrm{~Hz}, 1 \mathrm{H})$, 6.64-6.60 (m, $1 \mathrm{H}), 6.22(\mathrm{dd}, J=3.83,2.75 \mathrm{~Hz}, 1 \mathrm{H}), 5.74(\mathrm{~s}, 1 \mathrm{H})$, 3.87 (s, $3 \mathrm{H}), 3.81$ (s, $3 \mathrm{H}) \mathrm{ppm} .{ }^{13} \mathrm{C} \mathrm{NMR}\left(50.33 \mathrm{MHz}, \mathrm{CDCl}_{3}\right.$, TMS): $\delta=160.12,148.79,133.99,128.15,128.07,126.93,125.79$, $122.40,118.72,110.01,98.92,56.43,51.14 \mathrm{ppm}$. MS (EI): $\mathrm{m} / \mathrm{z}(\%)$ $=257(100)[\mathrm{M}]^{+}, 198(38), 164(85), 121(80) . \mathrm{C}_{15} \mathrm{H}_{15} \mathrm{NO}_{3}: \mathrm{C} 70.02$, H 5.88, N 5.44; found C 70.44, H 5.34, N 5.76.

\section{Acknowledgments}

This work was supported by grants from the Università di Torino and the Ministero dell\&apos;Università e della Ricerca (MIUR).

[1] a) L. DeLuca, Curr. Med. Chem. 2006, 13, 1-23; b) A. Tanitame, Y. Oyamada, K. Ofuji, M. Fujimoto, K. Suzuki, T. Ueda, H. Terauchi, M. Kawasaki, K. Nagai, M. Wachi, T. Yamagishi, J. Bioorg. Med. Chem. 2004, 12, 5515-5524; c) T. Uno, M. Takamatsu, Y. Inoue, Y. Kawahata, K. Iuchi, G. Tsukamoto, J. Med. Chem. 1987, 30, 2163-2169; d) H. Vanden Bossche, Curr. Top. Med. Mycol. 1985, 313-351; e) M. Ogata, H. Matsumhimizu, S. Kida, M. Shiro, K. Tawara, J. Med. Chem. 1987, 30, 1348-1354; f) A. Ruland, W. Reuther, E. Ammermann, E. H. Pommer, BASF, DE 31130215, 1983 [Chem. Abstr. 1983, 98, 215596].

[2] For a general review on cross-coupling reactions of azoles see: M. Schnürch, R. Flasik, A. F. Khan, M. Spina, M. D. Mihovilovic, P. Stanetty, Eur. J. Org. Chem. 2006, 3283-3307 and references cited therein.

[3] a) D. Ma, Q. Cai, Org. Lett. 2003, 5, 3799-3802; b) F. Y. Kwong, A. Klapars, S. L. Buchwald, Org. Lett. 2002, 4, 581584; c) C. Wolf, S. Liu, X. Mei, A. T. August, M. D. Casimir, J. Org. Chem. 2006, 71, 3270-3273; d) Z. Wang, W. Bao, Y. Jiang, Chem. Comm. 2005, 2849-2851. For a general review see: J. Lindley, Tetrahedron 1984, 40, 1433-1456.

[4] a) D. W. Old, M. C. Harris, S. L. Buchwald, Org. Lett. 2000, 2, 1403-1406; b) L. M. Alcazar-Roman, J. F. Hartwig, A. L. Rheingold, L. M. Liable-Sands, I. A. Guzei, J. Am. Chem. Soc. 2000, 122, 4618-4630; c) J. F. Hartwig, M. Kawatsura, S. I. Hauck, K. H. Shaughnessy, L. M. Alcazar-Roman, J. Org. Chem. 1999, 64, 5575-5580; d) M. Watanabe, M. Nishiyama, T. Yamamoto, Y. Koie, Tetrahedron Lett. 2000, 41, 481-483.

[5] A. Kiyomori, J.-F. Marcoux, S. L. Buchwald, Tetrahedron Lett. 1999, 40, 2657-2660.

[6] J. C. Antilla, A. Klapars, S. L. Buchwald, J. Am. Chem. Soc. 2002, 124, 11684-11688.

[7] J. C. Antilla, J. M. Baskin, T. E. Barder, S. L. Buchwald, J. Org. Chem. 2004, 69, 5578-5587.

[8] a) D. M. T. Chan, K. L. Monaca, R. P. Wanag, M. P. Winters, Tetrahedron Lett. 1998, 39, 2933-2936; b) A. P. Combs, S. Saubern, M. Rafalski, P. Y. S. Lam, Tetrahedron Lett. 1999, 40, 1623-1626; c) P. Y. S. Lam, S. Deudon, M. Averill, R. Li, M. Y. He, P. DeShong, C. G. Clark, J. Am. Chem. Soc. 2000, 122, 7600-7601; d) P. Y. S. Lam, C. G. Clark, S. Subern, J. Adams, K. M. Averill, D. M. T. Chan, A. Combs, Synlett 2000, 674676.

[9] P. Y. S. Lam, C. G. Clark, S. Saubern, J. Adams, M. P. Winters, D. M. T. Chan, A. Combs, Tetrahedron Lett. 1998, 39, 29412944. 
[10] W. W. K. R. Mederski, M. Lefort, M. German, D. Kux, Tetrahedron 1999, 55, 12757-12770.

[11] E. Alcade, I. Dinares, S. Rodriguez, C. Garcia de Miguel, Eur. J. Org. Chem. 2005, 1637-1643.

[12] S. Yu, J. Saenz, J. K. Srirangam, J. Org. Chem. 2002, 67, 16991702.

[13] P. Y. S. Lam, G. Vincent, C. G. Clark, S. Deudon, P. K. Jadhav, Tetrahedron Lett. 2001, 42, 3415-3418.

[14] a) J. P. Collman, M. Zhong, Org. Lett. 2000, 2, 1233-1236; b) J. P. Collman, M. Zhong, L. Zeng, S. Costanzo, J. Org. Chem. 2001, 66, 1528-11531.

[15] P. Y. S. Lam, G. Vincent, D. Bonne, C. Clark, Tetrahedron Lett. 2003, 44, 4927-4931.

[16] S. Dalili, A. K. Yudin, Org. Lett. 2005, 7, 1161-1164.

[17] A. Y. Labedev, V. V. Izmer, D. N. Kazyul\&apos;kin, I. P. Beletskaya, A. Z. Voskoboynikov, Org. Lett. 2002, 4, 623-626.

[18] M. Taillefer, A. Ouali, B. Renard, J.-F. Splinler, Chem. Eur. J. 2006, 12, 5301-5313.
[19] M. Movassaghi, A. E. Ondrus, J. Org. Chem. 2005, 70, 86388641.

[20] P. Balma Tivola, A. Deagostino, C. Prandi, P. Venturello, Org. Lett. 2002, 4, 1275-1277.

[21] a) M. Schlosser, J. Organomet. Chem. 1967, 8, 9-16; b) M. Schlosser, Mod. Synth. Methods 1992, 6, 227-271; c) A. Mordini, in: Advances in Carbanion Chemistry (Ed.: V. Snieckus), JAI Press, Inc., Greenwich, CT, 1992; vol. 1, pp. 1-45; d) M. Schlosser, F. Faigl, L. Franzini, H. Geneste, G. Katsoulos, G. Zhong, Pure Appl. Chem. 1994, 66, 1439-1446; e) L. Lochmann, Eur. J. Inorg. Chem. 2000, 1115-1126.

[22] D. M. T. Chan, Tetrahedron Lett. 1996, 37, 9013-9016.

[23] A. Klapars, J. C. Antilla, X. Huang, S. L. Buchwald, J. Am. Chem. Soc. 2001, 123, 7727-7729.

[24] T. D. Quach, R. Batey, Org. Lett. 2003, 5, 4397-4400.

Received: October 4, 2006 Published Online: January 17, 2007 